Journal of Engineering and Applied Sciences 14 (18): 6764-6768, 2019

ISSN: 1816-949X

(C) Medwell Journals, 2019

\title{
Segregation Process Control While Forming a Technogenic Deposit
}

\author{
Kaerbek Rafkatovich Argimbaev \\ Saint-Petersburg Mining University, St. Petersburg, 21 Line V.O., 2, 199106 Saint-Petersburg, \\ Russia Federation,Russia diamond-arg@mail.ru
}

\begin{abstract}
Today, particle segregation in tailing dumps occurs naturally and is not controlled. This fact makes difficult to deliniate areas where it is cost-effective and expedient to process mill tailings. Control over segregation process during the technogenic deposit formation solves a plenty of problems during the tailing dump reprocessing. Namely, it gives a clear view of the valuable component content in a particular processed site, identifies areas that are promising for use in various industries, reduces their downblending and significantly increases the mining operation safety. During this study, a device and a basic layout of the plant intended to manage particle segregation during the technogenic deposit formation were worked out. Laboratory studies of the polydisperse mixture segregation have shown that application of this plant resulted in increasing separation performance for polydisperse mixtures during their stacking in different positions (horizontal and vertical) and decreasing the temperature effect on segregation. The dependences were revealed for the distribution of axial and tangential components of the flow speed over the pipe cross-section as well as the effect of solid swirling on the change of the flow structure and separation efficiency.
\end{abstract}

Key words: Technogenic deposit segregation, speed, computer simulation, device, fraction, polydisperse

\section{INTRODUCTION}

Involvement of a yet formed technogenic deposit into its processing is of great environmental and economic importance. When calculating economic efficiency, it is necessary to take into account not only additional product output but also the reduction of the costs for creating and operating tailing dumps, waste storage, prevention or diminishing the environmental pollution damage caused by dressing mill wastes (Pashkevich and Petrova, 2014; Argimbaev, 2018).

By reducing the content of valuable component in tailings even by basic points, it is possible to get a significant effect due to the huge production quantities and cost reduction due to decreasing the cost of crushing and grinding operation which accounts for $40-50 \%$ of the total cost during the normal ore processing (Kholodnyakov and Argimbayev, 2014a, b; Argimbaev and Kholodjakov, 2016). When processing tailings, these items of expenditure will disappear as tailings will be already crushed and broken down for almost any processing method.

Allocation of the boundaries for promising, cost-effective zones and ones expedient for processing in the technogenic deposit being formed is a topical problem in modern science (Fomin and Kholodjakov, 2012; Argimbaev and Kholodjakov, 2015; Gavrishev et al., 2016). To solve this problem, it is necessary to complete the following tasks: to study the effect of spiral solid swirling on the polydisperse flow structure in a pipeline, to determine process parameters of the laboratory plant intended to control segregation while forming a technogenic deposit, to manufacture a laboratory plant; to carry out laboratory study of the polydisperse mixture segregation, to reveal distribution dependences for axial and tangential components of flow speed in a pipeline cross-section, the effect of solid swirling on the flow structure change as well as separation efficiency.

\section{MATERIALS AND METHODS}

During the research, a modern approach was used to solve the set problems-complex study analytical method comprising assessment of the segregation control prerequisites when forming a technogenic deposit.

Computer simulation method comprising simulation of influence of spiral solid swirling parameters on the flow structure (while keeping the dynamic similarity by Reynolds number).

A lab technique comprising physical simulation of controlled segregation and the study of the polydisperse mixture behaviour during the technogenic deposit formation.

Mathematical analysis of the obtained laboratory results. Synthesis of particular research results in different fields of science. 


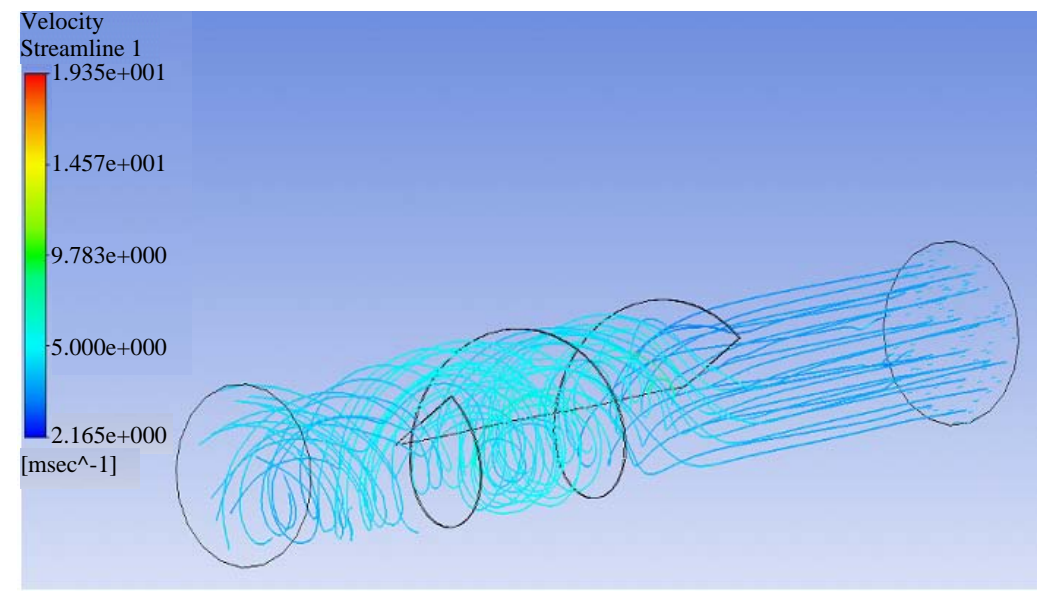

Fig. 1: Computer simulation of the slurry stream with the spiral solid swirling inserted into the pipeline

\section{RESULTS AND DISCUSSION}

The research performed has shown that it was possible to control segregation process during the technogenic deposit formation by inserting solid swirling into a pipeline. It facilitated the increase of the tailing stream speed, changing the motion trajectory as well as artificial segregation of mill tailings.

Spiral movement formation using the stream solid spiral swirling allows mill tailing conveyance at a significantly lower stream speed without precipitation of heavy/solid particles in a pipeline. It also forms favourable conditions for their segregation.

The effect of spiral solid swirling on the stream structure in a pipeline was studied using the created plant (while keeping the dynamic similarity by Reynolds number) as well as computer simulation using ANSYS CFX Software (license No. is 75458657 , proprietor is St.-Petersburg Mining University) (Fig. 1).

Based on the computer model (Fig. 1), we observed the transformation of laminar motion into turbulent one that allowed creating a segregation zone for technogenic material.

The device model and a basic layout of the segregation control plant (Fig. 2) were worked out on the basis of the description and computer simulation.

The presented model or the segregation process control during the technogenic deposit formation facilitates the polydisperse flow speed, the change of motion trajectory and artificial segregation.

The model operates in the following way: polydisperse mixture conveyed by the pipeline is supplied into the pipe section containing cylindrical device. When passing this section, the mixture enters the solid spiral guiding device where laminar motion transforms into

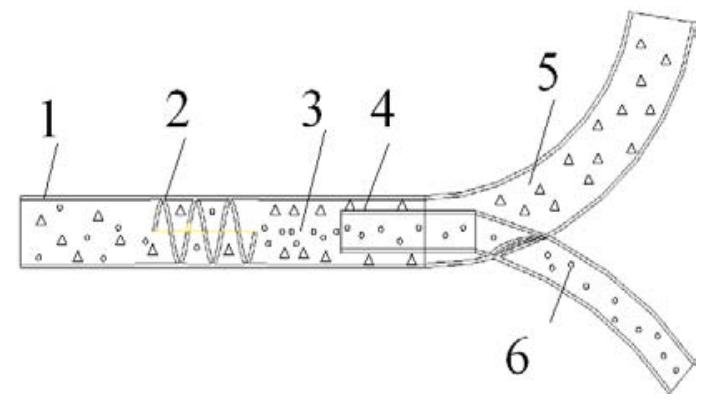

Fig. 2: Cross section of the segregation control device; 1 cylindrical body; 2 solid spiral guiding device; 3 zone of the mixture turbulent motion (layer splitting); 4 layer splitter; 5 coarse (rich) fraction and 6 fine (bare) fraction

turbulent one. Leaving this device, the mixture enters the turbulent motion area (layer splitting) where particle segregation takes place under effect of axial, tangential speed and centrifugal forces. During segregation, coarse (rich) fraction conveys via. the bigger pipe while fine (bare) fraction is transported by the smaller one. Pipe branching with coarse (rich) and fine (bare) fractions may have different design.

The segregated fractions in the layered flow are fed into the layer splitter which in turn, guides them into different directions.

Basic parameters defined during the computer simulation allowed to assemble a pilot plant used for the laboratory study of the spiral insert effect on the segregation of technogenic raw material (Fig. 3).

A Makita centrifugal pump with conveyance capacity of $15 \mathrm{~m}^{3} / \mathrm{h}$ was used to feed technogenic raw material. It was able to transport particles up to $35 \mathrm{~mm}$ in diameter (Fig. 4). 

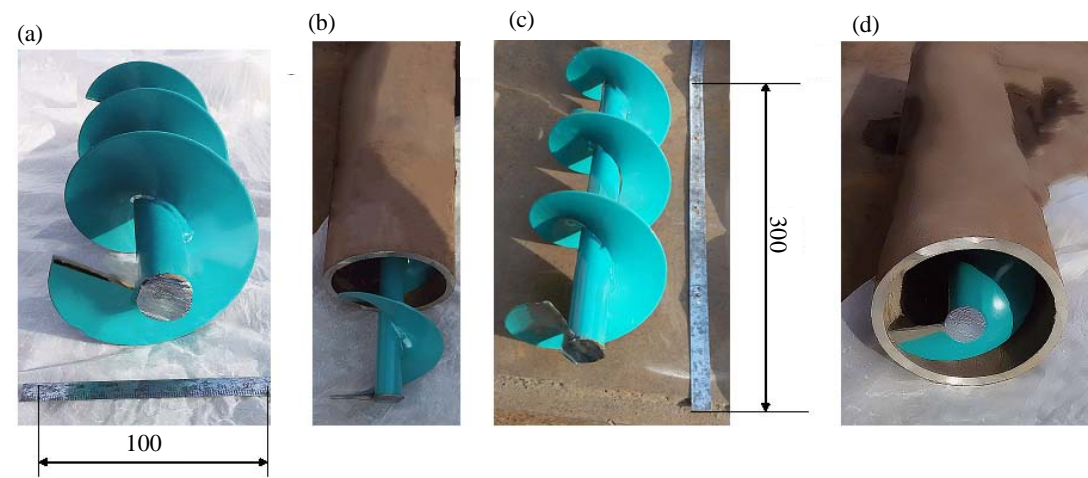

Fig. 3: Pilot plant manufacturing: a-d) plant assembly process

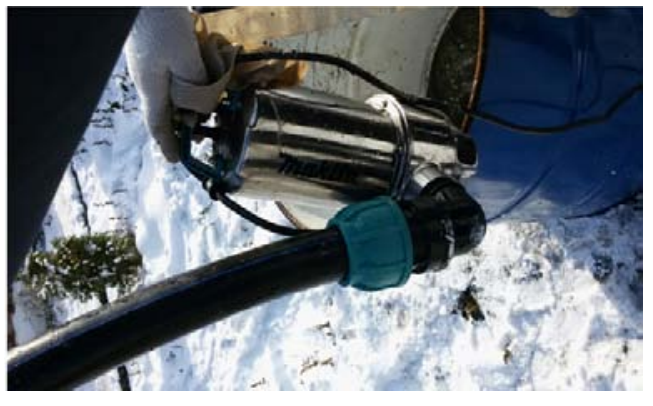

Fig. 4: Makita PF1110 centrifugal pump

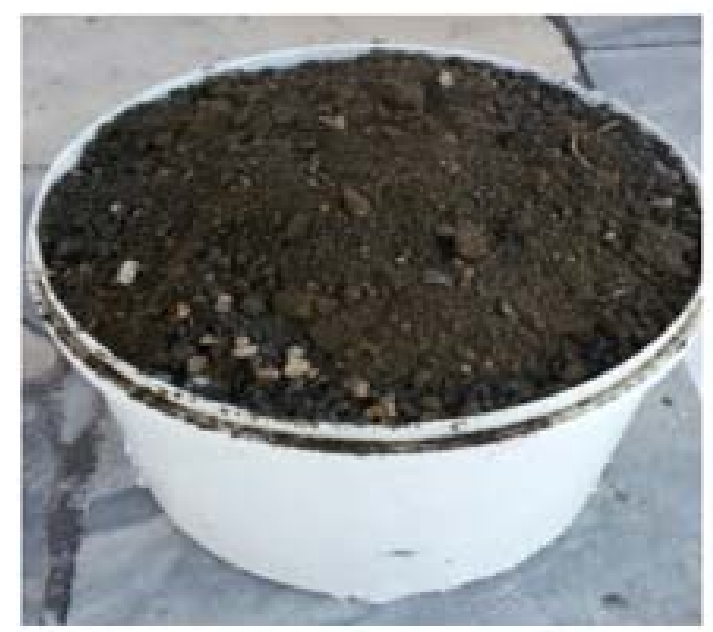

Fig. 5: Basic initial material

Experiments were carried out using different polydisperse materials formed on the basis of the slurry taken from slurry damps of Magnitogorsk mining and processing works (Fig. 5).

During the laboratory and theoretical study, speed characteristics were measured for the polydisperse mixture when changing swirling pitch. Experimental and theoretical results are given in Table 1 with the given values:

$$
\overline{\vartheta_{\mathrm{T}}}=\frac{\vartheta_{\mathrm{T}}}{\vartheta_{\mathrm{L}}} ; \overline{\vartheta_{\mathrm{o}}}=\frac{\vartheta_{\mathrm{o}}}{\vartheta_{\mathrm{L}}} ; \overline{\mathrm{D}}=\frac{\mathrm{h}}{\mathrm{D}}
$$

Where:

$$
\begin{aligned}
\vartheta_{\mathrm{T}}=\vartheta_{\mathrm{L}} \cdot \cos \beta= & \text { The axial }(\text { tangential) component of the } \\
& \text { flow local speed }\left(\vartheta_{\mathrm{L}}\right) \\
\vartheta_{\mathrm{o}}=\vartheta_{\mathrm{L}} \cdot \sin \beta= & \text { The speed tangential component }\left(\vartheta_{\mathrm{L}}\right) \\
\beta & \text { The angle between the pipeline axis and } \\
& \text { direction }\left(\vartheta_{\mathrm{L}}\right) \\
= & \text { The pipeline diameter } \\
= & \text { The measuring point ordinate relatively } \\
& \text { the inner surface of the horizontal pipe } \\
& \text { lower wall }
\end{aligned}
$$

To swirl the flow in horizontal straight-line pipe sections, spiral solid inserts to segregate the materials conveyed are quite efficient and optimal (by economic considerations).

Speed tangential component is zero if there is no spiral solid swirling in the pipe. Speed axial component is the highest in the flow central part and sharply decreases when measurement points approach the pipeline walls (Fig. 6).

If a spiral solid insert installed in the pipe, polydisperse mixture swirls and becomes nonaxisymmetric (shifted to any side depending on the swirling direction). Swirling speed in the lower part of the pipeline wall is:

$$
\vartheta_{\mathrm{o}}=0.2 \cdot \vartheta_{\mathrm{L}}
$$

within the spiral insert. Beyond the source of the spiral solid swirling, the flow twisting degree gradually decreases and the effect of the spiral insert becomes minimal at a distance of 50-55D (Table 2). Tangential speed near the pipe walls facilitates efficient suspending and maintaining solid particles in the layered flow. 
Table 1: Slurry grain size composition

\begin{tabular}{|c|c|c|c|c|c|}
\hline \multirow[b]{2}{*}{ Fineness $(\mathrm{mm})$} & \multicolumn{5}{|l|}{ Sample index } \\
\hline & Sample No. $1(\gamma(\%))$ & Sample No. $2(\gamma(\%))$ & Sample No. $3(\gamma(\%))$ & Sample No. $4(y(\%))$ & Sample No. $5(\gamma(\%))$ \\
\hline$-20.0+15.0$ & 1.42 & 0.13 & 1.89 & 0.96 & 0.72 \\
\hline$-15.6+8.0$ & 4.54 & 3.81 & 6.57 & 5.64 & 6.24 \\
\hline$-8.0+4.0$ & 5.14 & 7.88 & 8.61 & 6.85 & 9.25 \\
\hline$-4.14+3.1$ & 8.01 & 7.05 & 9.24 & 10.54 & 8.65 \\
\hline$-3.1+1.0$ & 7.12 & 11.45 & 12.50 & 12.67 & 9.52 \\
\hline$-1.0+0.05$ & 55.00 & 60.47 & 48.15 & 55.60 & 56.02 \\
\hline-0.05 & 18.77 & 9.21 & 13.04 & 7.74 & 9.60 \\
\hline Total output (\%) & 100.00 & 100.00 & 100.00 & 100.00 & 100.00 \\
\hline
\end{tabular}

Table 2: Effect of spiral solid swirling on the alternation of flow structure and separation efficiency

\begin{tabular}{lccc} 
Parameters & $\begin{array}{c}\text { Axial speed } \overline{\vartheta_{\circ}} \\
\text { (without a spiral insert) }\end{array}$ & $\begin{array}{c}\text { Axial speed } \overline{\bar{\vartheta}_{\circ}} \\
\text { (an axial insert with 1 }=\text { D pitch) }\end{array}$ & $\begin{array}{c}\text { Axial speed } \overline{\vartheta_{\circ}} \\
\text { (an axial insert with 1 } \approx 20 . \mathrm{D} \text { pitch) }\end{array}$ \\
\hline $\begin{array}{l}\text { Normalized flow speed }(\mathrm{m} / \mathrm{sec}) \\
\text { Separation efficiency for fraction }\end{array}$ & 1.02 & 1.18 & 1.05 \\
$-3.1+0$ at the splitter set point $0.5^{*} \mathrm{D}(\%)$ & 15.00 & 88.00 & 25.00 \\
\hline
\end{tabular}

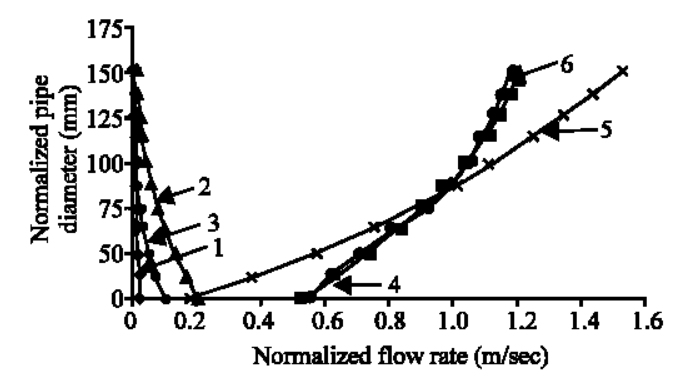

Fig. 6: Distribution of speed axial and tangential components for the flow in the pipe cross section; 1 normalized tangential speed $\overline{\theta_{0}}$ (without a spiral insert); 2 normalized tangential speed $\overline{\vartheta_{\circ}}$ (a spiral insert with $1=\mathrm{D}$ pitch); 3 normalized tangential speed $\overline{\vartheta_{\circ}}$ (a spiral insert with $1 \approx 20$.D pitch); 4 normalized axial speed $\overline{\theta_{\mathrm{T}}}$ (without a spiral insert); 5 normalized axial speed $\overline{\vartheta_{\mathrm{T}}}$ (a spiral insert with $1=$ D pitch), 6 normalized axial speed $\overline{\theta_{\mathrm{T}}}(\mathrm{a}$ spiral insert with pitch $1 \approx 20$.D)

Installation of a solid swirling device increases the speed axial component on average by $25 \%$ in the central part due to its decrease in the pipe near-wall area.

Thus, the change of the slurry speed parameters facilitates particle segregation. The high axial speed promotes reliable retention of fine and light-weight particles in the flow core and prevention against their sedimentation into the near-wall areas. Segregation results are shown in Fig. 7.

The least square method of experimental data processing allowed obtaining the dependence that helped to define the local averaged axial speed in the near-bottom part of twisted flow:

$$
\vartheta_{\text {T.O }}^{\prime}=0.4 . \vartheta_{\text {T.O. }} \cdot \frac{1^{0.2}}{D^{0.2}}
$$

(a)

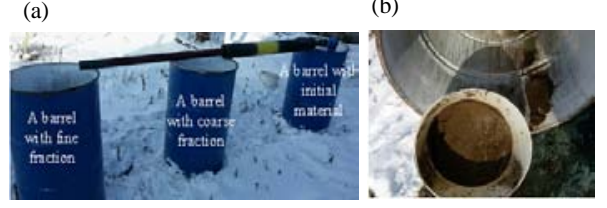

(c)

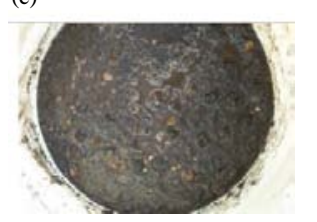

Fig. 7: Segregation results for technogenic raw material; a) General appearance of the segregation plant; $b$ ) Fine fraction and c) Coarse fraction

Where:

$\vartheta_{\mathrm{T} . \mathrm{O}}=$ The flow speed averaged component in the near-bottom area of the horizontal straightline pipe without a perturbance source

$\mathrm{m} / \mathrm{sec} ; 1=$ The distance from the spiral insert end to the measurement point $(\mathrm{m})$

\section{CONCLUSION}

Solid particles conveyed along the straight section near the lower part of pipe wall are involved into spiral motion from the very beginning of the vortex motion in the helical fins. For this type of the carrying medium motion, solid inclusions tend to locate in the layered stream in the area of both tangential and increased axial speed values.

Thus, flow swirling at remaining average conveyance speed allow's to separate the flow by particle size and density with separation efficiency of $88 \%$ for fine fraction $-3.1+0 \mathrm{~mm}$ (Table 2) at swirling pitch $1=\mathrm{D}$. 


\section{REFERENCES}

Argimbaev, K.R. and H.A. Kholodjakov, 2015. Erection methods and constructions of primary tailing dike. Intl. J. Ecol. Develop., 30: 47-54.

Argimbaev, K.R. and H.A. Kholodjakov, 2016. Tailings development and their utilization in the national economy. Int. J. Ecol. Dev., 31: 94-100.

Argimbaev, K.R., 2018. Research of the dynamics of cryogenic processes in man-made deposits presented by dressing plant waste. J. Eng. Appl. Sci., 13: 1621-1623.

Fomin, S.I. and G.A Kholodjakov, 2012. Opencast mining parameters evaluation at preliminary study of mining project. Sci. Rep. Resour. Issues, 1: 382-386.
Gavrishev, S.E., K.V. Burmistrov, S.N. Kornilov and N.G. Tomilina, 2016. Evaluation of transportation flow charts with open-pit hoisting systems in open pit/underground mining. Gornyi Zh., 5: 41-47.

Kholodjakov, H.A. and K.R. Argimbaev, 2014b. Waste storage feasibility of concentrating mill in overburden dumps. World Appl. Sci. J., 30: 738-740.

Kholodnyakov, G.A. and K.R. Argimbayev, 2014a. The choice and substantiation of the technological parameters of tailing formation in an overburden dump body. Scientific Bull. National Mining Univ., 2: $50-57$.

Pashkevich, M. and T. Petrova, 2014. Ecological and geochemical features of technogenic massif transformation at Severonickel (Russia). Intl. J. Ecol. Dev., 29: 110-114. 